\title{
Oligodendroglial Cell Death in Jimpy Mice: An Explanation for the Myelin Deficit
}

\author{
P. E. Knapp, R. P. Skoff, and D. W. Redstone \\ Department of Anatomy, Wayne State University School of Medicine, Detroit, Michigan 48201
}

\begin{abstract}
Neuroglial cell death was investigated in 3 white matter tracts of jimpy and normal mice. In normal animals, glial death during development ranged from 0.5 to $2.7 \%$ of the total glial population. The number of dying glial cells was significantly higher in jimpy animals at times corresponding with oligodendroglial proliferation and the onset of myelination in each tract. At certain ages, over $10 \%$ of the glial population were pyknotic at the lightmicroscopic level. Dying glial cells that were identified ultrastructurally presented the characteristics of oligodendrocytes. Premature death of oligodendrocytes presents a simple explanation for the gross deficits of myelin in jimpy animals. The shortened life span of the jimpy oligodendrocyte may preclude the elaboration of a normal myelin sheath. The jimpy model may prove to be a valuable tool in delineating a role for normal neuroglial cell death during the development of the nervous system.
\end{abstract}

Cell death has been shown to play a significant role in the structuring of many tissues during development (Glücksmann, 1951). Neuronal cell death has been examined extensively in this regard. Elimination of a portion of the neuronal population is a normal developmental process helping to determine the ultimate size of neuronal populations and their relationships to target cells (Cowan et al., 1984; Hamburger and Oppenheim, 1982). Neuroglial cell death may also contribute to the development of the nervous system, perhaps by establishing normal ratios of glial cell types and by refining associations between neurons and glial cells themselves. The death of neuroglial cells during development has been described in only a few studies (Korr, 1980; Smart and Leblond, 1961; Sturrock, 1979), and no functional significance has been attached to this event. We have recently reported an increased incidence of glial cell death in the jimpy mouse (Knapp and Skoff, 1984). Jimpy (jp) is a sexlinked mutation, which results in severe hypomyelination in the CNS of affected male mice (Sidman et al., 1964). The high level of glial cell death in the jp mouse may help us to understand the normal role of neuroglial death in developing nervous systems.

In this paper we use light-microscopic techniques to show that the amount of glial death in developing jp CNS tissues can be greater than $10 \%$ of the total glial population, and greater than 10 times that of normal tissues. Using ultrastructural techniques we further establish that oligodendrocytes are primarily affected. The premature and selective death of the oligodendrocyte population helps to explain the well documented reduction

Received June 3, 1985; revised Mar. 10, 1986; accepted Apr. 1, 1986.

This work was supported by NIH Grant NS 15338. We wish to thank Dr. William Bartlett and Dr. Joyce Benjamins for their helpful suggestions in the preparation of the manuscript.

Correspondence should be addressed to Dr. Pamela E. Knapp, Department of Anatomy, Wayne State University, 540 E. Canfield Ave., Detroit, MI 48201

Copyright (C) 1986 Society for Neuroscience $0270-6474 / 86 / 102813-10 \$ 02.00 / 0$ of oligodendrocyte numbers in jp animals (Kraus-Ruppert et al., 1973; Meier and Bischoff, 1975; Skoff, 1976) in the face of their increased uptake of tritiated thymidine (Skoff, 1982). Most importantly, the early death of jp oligodendrocytes indicates that the cell type is defective and probably unable to perform its normal task of making and maintaining a myelin sheath.

\section{Materials and Methods}

Mice used in these experiments were males bred in our laboratory by mating jp carrier females (Tajp/ ${ }^{++}$) with tabby males $\left(\mathrm{Ta}^{+} / \mathrm{Y}\right)$ obtained from the Jackson Laboratories (Bar Harbor, ME). Male offspring that receive the jp gene show a severe deficit in myelination of the CNS (Sidman et al., 1964). Young jimpy males (Tajp/Y) were identified by the lack of myelin in spinal cord sections at the light-microscopic level. The tabby gene (Ta), which is closely linked to the jp gene, produces characteristic fur markings that assist in the selection of jp animals. Jimpy animals older than $10 \mathrm{~d}$ were identified by their characteristic tremors. Normal control animals $(++/ Y)$ were identified by their normally myelinated spinal cords, lack of tremors, and wild-type (non-Ta) fur markings.

Mice were anesthetized with chloral hydrate and sacrificed by intracardiac perfusion at $4,7,11-12,16,20$, and $22 \mathrm{~d}$ after birth. The perfusate consisted of $2 \%$ glutaraldehyde, $2 \%$ paraformaldehyde, and $0.01 \% \mathrm{CaCl}$ in $0.1 \mathrm{M}$ phosphate buffer, $\mathrm{pH} 7.2$. Following perfusion, the intracranial portion of the optic nerve, the upper brachial segment of the spinal cord, and a midsagittal section of the corpus callosum at the level of the hypothalamus were dissected out. The tissue was placed in fixative and refrigerated overnight. The following day tissues were washed in $0.1 \mathrm{M}$ phosphate buffer, osmicated for $90 \mathrm{~min}$, dehydrated in graded ethanols, and placed in propylene oxide. The tissue was infiltrated overnight in a $50 \%$ solution of Araldite in propylene oxide, embedded in fresh Araldite, and polymerized at $56^{\circ} \mathrm{C}$ for $60 \mathrm{hr}$.

For light microscopy, $1 \mu \mathrm{m}$ sections were stained with an aqueous solution of toluidine blue. For electron microscopy, thin sections were stained with uranyl acetate and lead citrate. Thick sections were taken immediately preceding thin sections in order to facilitate the identification of individual cells in both light and electron micrographs.

Thick sections were used to quantify numbers of apparently normal and dying glia. Cells were counted in hemisections of spinal cord white matter and complete transverse sections of optic nerve and corpus callosum. In these areas there are no neuronal cell bodies, and glial cells were easily identified by typical nuclear and cytoplasmic features (Mori and Leblond, 1970). Microglia and endothelial cells were not included in the cell counts. The classification of a cell as dying involved a qualitative judgment on our part. The majority of cells included in this category were clearly morbid, consisting of a dense mass of cellular debris (Fig. 2). The identification of cells in less advanced stages of detcrioration, but still indexed as dying, was principally bascd on findings of abnormality in the distribution and density of nuclear chromatin. Changes in nuclear structure seemed to precede those in the cytoplasm and were more noticeable than cytoplasmic changes at light-level magnifications. Given the limited resolution of the light microscope, it seems probable that the percentages of dying glia that we calculated actually underestimate the severity of cell death in the jp animal. All 3 brain regions were examined at the ultrastructural level. Figures were largely drawn from $20 \mathrm{~d}$ spinal cord sections because of the large number of dying cells in this area. Statistical analyses included SEM and 2-tailed $t$ tests to establish differences between jp and control animals. 

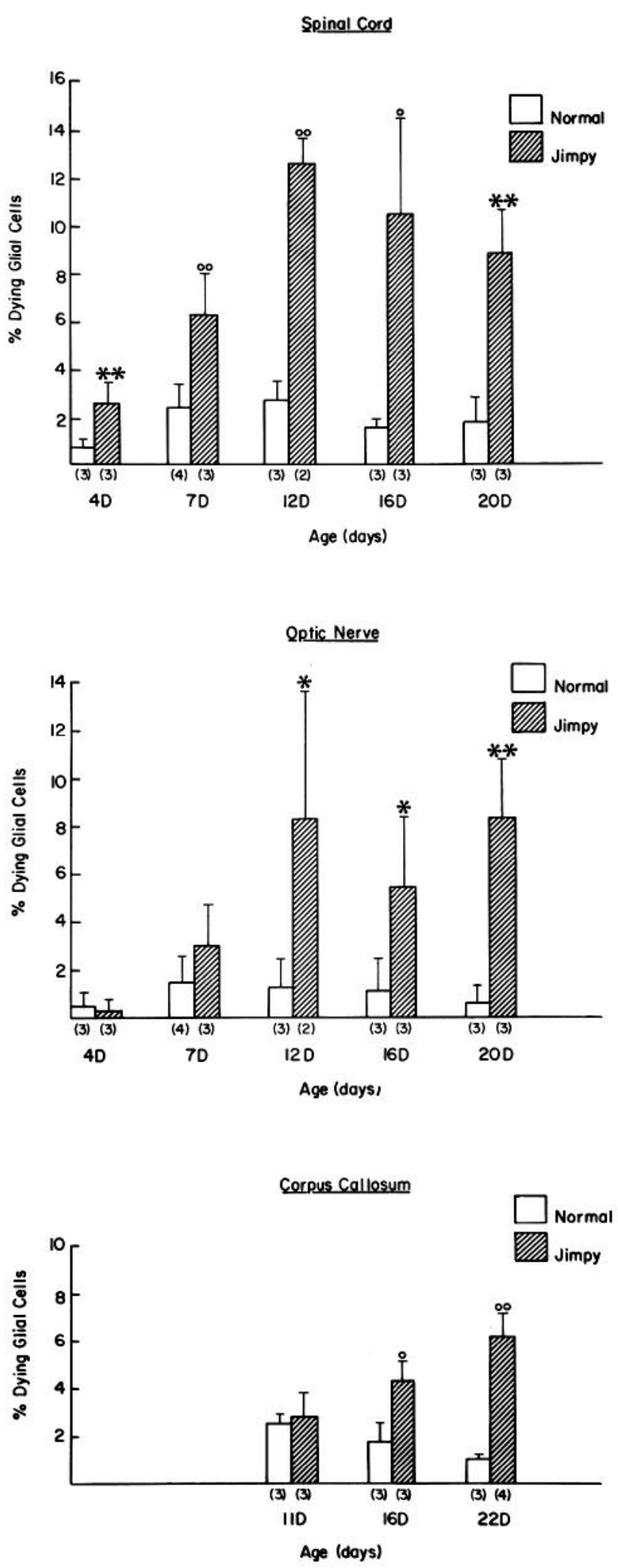

Figure 1. Percentages of dying glial cells in normal and jp male mice. Bars, SEM. The number of samples is given in parentheses. ${ }^{*} p<0.05$, ${ }^{\circ} p<0.01,{ }^{* *} p<0.025,{ }^{\infty} p<0.001$.
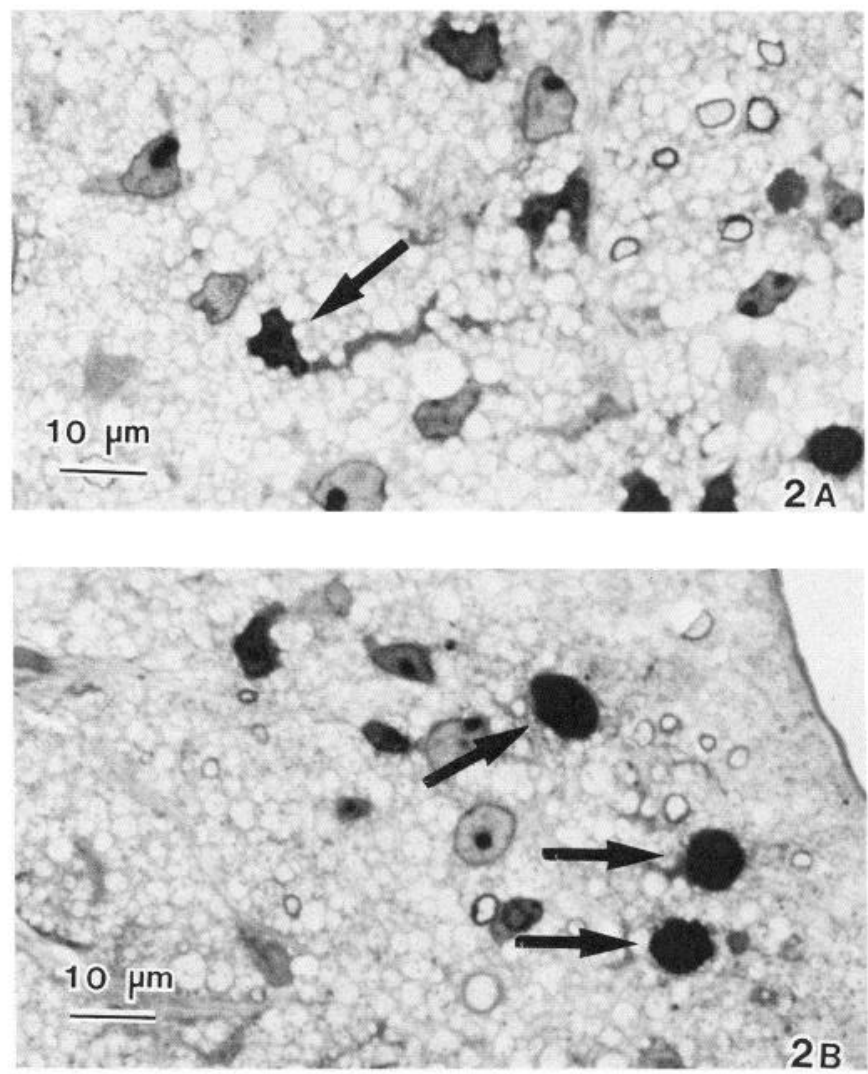

Figure 2. Twenty day jp mouse spinal cord. $\times 950$. A, Dying cell (arrow) in early stages of degeneration. $B$, Numerous dying cells in advanced stages of degeneration (arrows). Note the paucity of myelinated axons.

\section{Results}

\section{Light-microscopic quantification}

Glial death in normal tissues ranged between 0.5 and $2.7 \%$ of the total glial population (Fig. 1). The percentages of dying glial cells in the CNS of jp animals depended both upon the region of the brain and the age of the animal. The absolute numbers of dying and total glial cells are given in Table 1 .

In the 4-d-old mouse, spinal cord myelination has already begun. Spinal cords of jp animals had percentages of dying glia that were significantly higher than in control animals at all ages studied (4-20 d postnatal). The jp mutant shows a 4-fold increase in glial cell death at $4 \mathrm{~d}$ of age. Differences between jp and control animals became even greater at older ages. The highest percentage of glial cell death we observed in the jp spinal cord was $12.6 \%$ at day 12 . Although the quantification was done on cell counts in the white matter, large numbers of dying cells were also seen in the gray matter of the cord.

Myelination in the optic nerve begins at a later time than in the spinal cord, starting between days 5 and 7 (Kuwabara, 1975; Tennekoon et al., 1980). Percentages of dying glial cells in jp optic nerves were similar to controls at day 4 and started to increase by $7 \mathrm{~d}$ of age. By day 12, and thereafter, levels were significantly higher in jp animals. At day 20 there was a 12 -fold difference between jp and normal optic nerves and the highest percentage $(8.4 \%)$ of dying glia was observed.

The first myelin sheaths in the mouse corpus callosum (CC) were seen at day 11 , although the phase of rapid myelination occurs between 14 and $45 \mathrm{~d}$ (Sturrock, 1980). We studied the $\mathrm{CC}$ at 3 time points during development: 11,16 , and 22 days. At day 11,jp and control sections had equivalent numbers of 


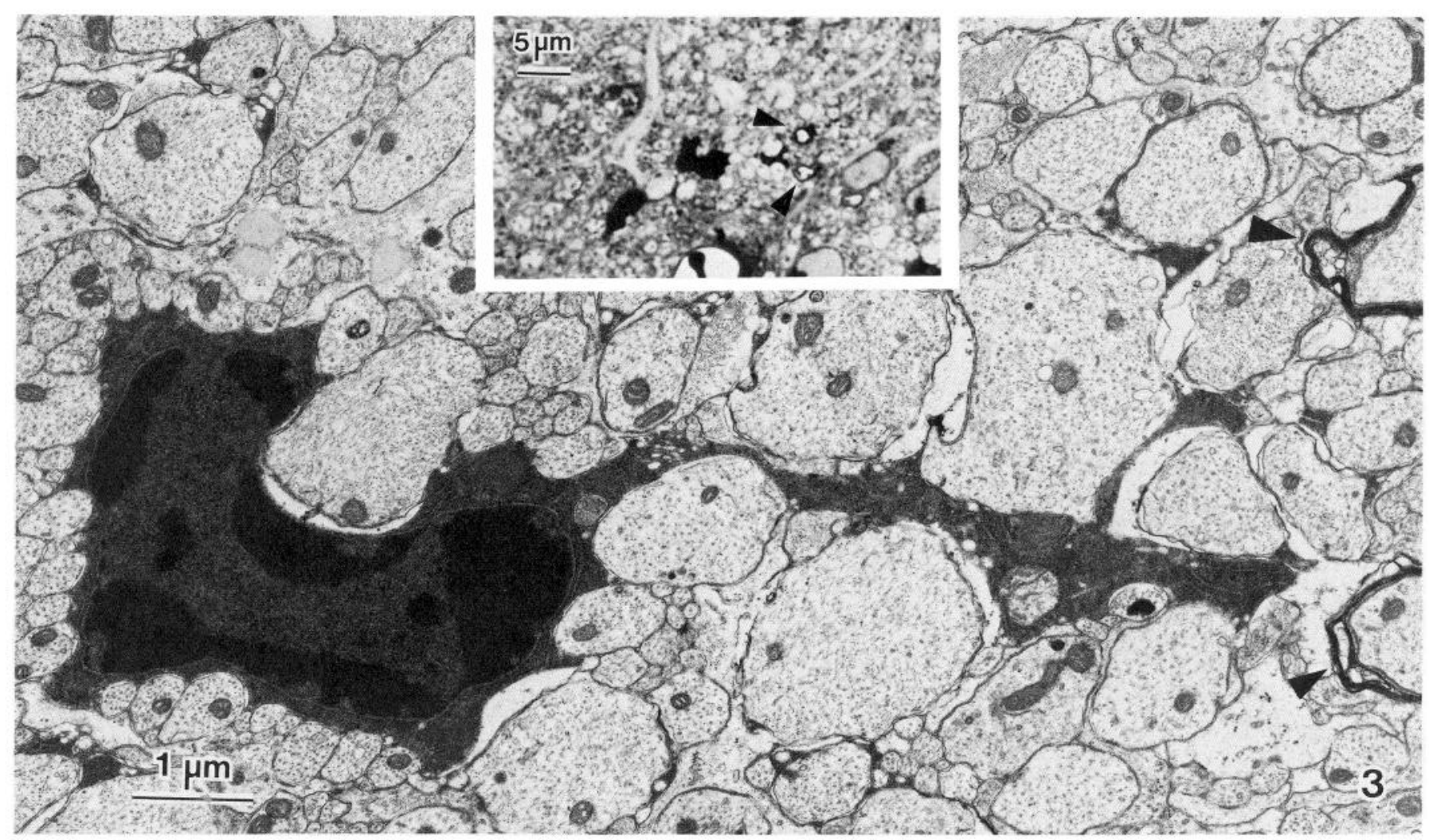

Figure 3. Electron micrograph of a dying cell in 20 -d-old jp mouse spinal cord. $\times 16,000$. Inset, Corresponding cell in an adjacent thick section. $\times 1400$. Arrowheads indicate 2 myelinated axons that are found in both sections; the remainder of the axons are unmyelinated.

dying glia. By day 16 the jp CC had a statistically higher percentage of dying cells, a difference that increased to 6 -fold by day 22 .

Representative light micrographs illustrating typical dying glial cells in jp are included (Fig. 2). Some of the dying cells have long cytoplasmic processes, but most are spherical and condensed. There were no gross morphological differences between dying cells in normal versus jp tissues.

\section{Electron-microscopic analysis}

Alternating thick and thin sections were initially used to confirm the status of the cells that appeared to be dying at the light level. Light and electron micrographs of the same cell taken from adjacent thick and thin sections of a $20 \mathrm{~d}$ jp spinal cord are shown (Fig. 3). The dying cell, which is unidentifiable at the low resolution of the light microscope, shows features of an oligodendrocyte when viewed ultrastructurally. Electron microscopy was used to identify conclusively the type(s) of dying glial cells.

Cells in early stages of necrosis could be identified as immature oligodendroglia by cytoplasmic features characteristic of this cell type (Mori and Leblond, 1970; Skoff et al., 1976). The cells shown in Figures 5-9 represent the likely continuum of stages through which glial cells in the jp mutant proceed during the course of their death. In contrast, an oligodendrocyte from a $20 \mathrm{~d}$ jp spinal cord, similar in morphology to myelinating oligodendrocytes in normal tissues, is seen in Figure 4. The features of this cell type distinguishing it from astroglia and microglia are: the overall density of the cytoplasm and nucleus; the elongated, thin strands of endoplasmic reticulum; numerous mitochondria; and stacks of Golgi apparatus. The nucleus is typically eccentric with chromatin distributed around the nuclear membrane. Several microtubules are present in this cell but are difficult to resolve because of the density of the cytoplasm. It appears to have connections with 2 axons.

The first sign of necrosis occurs in the nucleus where the chromatin is polarized (Fig. 5). Mitochondria and Golgi apparatus are numerous and normal in appearance. The nucleus retains its eccentric position. A cell in a more necrotic stage

Table 1. Average numbers of dying glia per total glial cells in cross sections of optic nerve and corpus callosum or hemisections of spinal cord

\begin{tabular}{|c|c|c|c|c|c|c|}
\hline \multirow[b]{2}{*}{ Day } & \multicolumn{2}{|c|}{ Spinal cord } & \multicolumn{2}{|c|}{ Optic nerve } & \multicolumn{2}{|c|}{ Corpus callosum } \\
\hline & Normal & Jimpy & Normal & Jimpy & Normal & Jimpy \\
\hline 4 & $\begin{array}{l}\frac{2.00}{350} \\
(n=3)\end{array}$ & $\begin{array}{l}\frac{9.67}{391} \\
(n=3)\end{array}$ & $\begin{array}{l}\frac{0.40}{85.4} \\
(n=5)\end{array}$ & $\begin{array}{l}\frac{0.25}{83.5} \\
(n=4)\end{array}$ & - & - \\
\hline 7 & $\begin{array}{l}\frac{9.50}{435} \\
(n=4)\end{array}$ & $\begin{array}{l}\frac{25.3}{413} \\
(n=3)\end{array}$ & $\begin{array}{l}\frac{1.50}{101} \\
(n=4)\end{array}$ & $\begin{array}{l}\frac{3.67}{116} \\
(n=3)\end{array}$ & - & - \\
\hline $11-12$ & $\begin{array}{l}\frac{16.0}{626} \\
(n=3)\end{array}$ & $\begin{array}{l}\frac{68.5}{546} \\
(n=2)\end{array}$ & $\begin{array}{l}\frac{2.00}{141} \\
(n=3)\end{array}$ & $\begin{array}{l}\frac{12.5}{146} \\
(n=2)\end{array}$ & $\begin{array}{l}\frac{13.3}{577} \\
(n=3)\end{array}$ & $\begin{array}{l}\frac{15.0}{553} \\
(n=3)\end{array}$ \\
\hline 16 & $\begin{array}{l}\frac{5.33}{400} \\
(n=3)\end{array}$ & $\begin{array}{l}\frac{55.0}{453} \\
(n=3)\end{array}$ & $\begin{array}{l}\frac{1.67}{133} \\
(n=3)\end{array}$ & $\begin{array}{l}\frac{6.33}{121} \\
(n=3)\end{array}$ & $\begin{array}{l}\frac{8.67}{514} \\
(n=3)\end{array}$ & $\begin{array}{l}\frac{19.3}{452} \\
(n=3)\end{array}$ \\
\hline $20-22$ & $\begin{array}{l}\frac{8.00}{475} \\
(n=3)\end{array}$ & $\begin{array}{l}\frac{50.3}{576} \\
(n=3)\end{array}$ & $\begin{array}{l}\frac{1.00}{140} \\
(n=3)\end{array}$ & $\begin{array}{l}\frac{14.3}{169} \\
(n=3)\end{array}$ & $\begin{array}{l}\frac{4.20}{548} \\
(n=4)\end{array}$ & $\begin{array}{l}\frac{25.0}{408} \\
(n=4)\end{array}$ \\
\hline
\end{tabular}



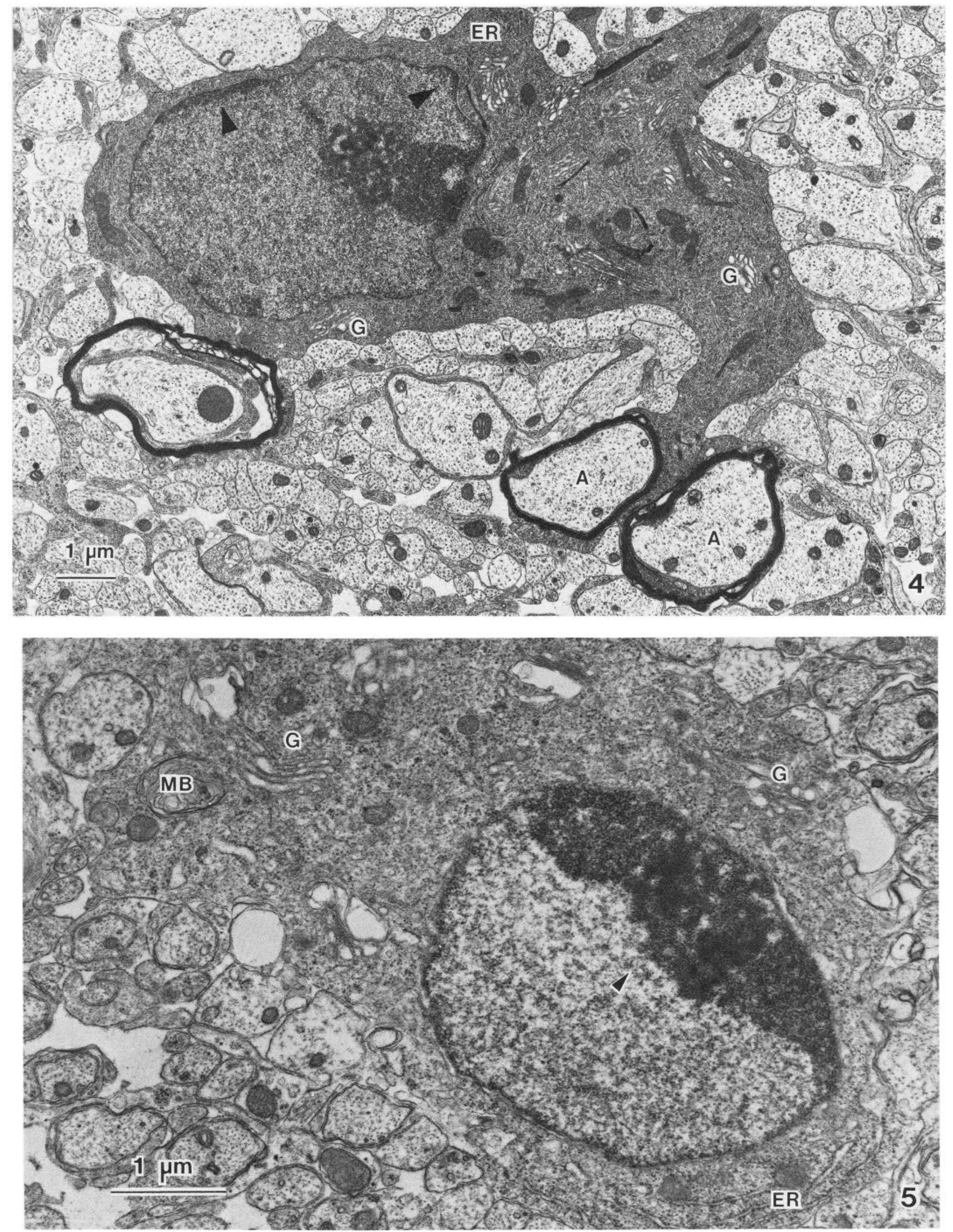


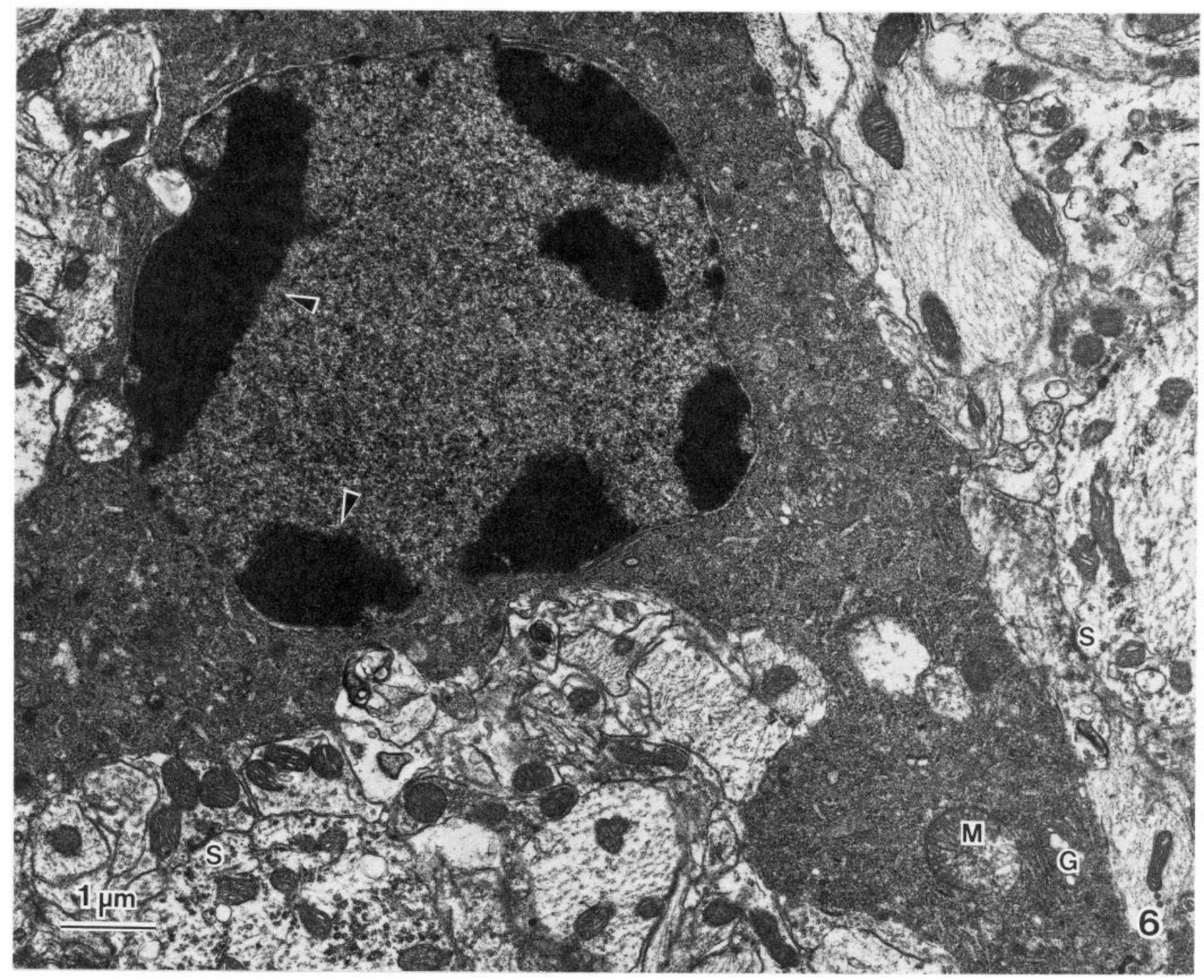

Figure 6. Twenty day jp mouse spinal cord. $\times 14,500$. A dying oligodendrocyte located in the gray matter of the cord. The nuclear chromatin is present in extremely condensed patches (arrows), the cytoplasm assumes a grainy texture, and organelles are in a less orderly arrangement than in normal cells of this type ( $G$, Golgi apparatus; $E R$, endoplasmic reticulum).

(Fig. 6) has nuclear chromatin present in several abnormally condensed patches at the periphery. Numerous mitochondria, Golgi apparatus, and the darker cytoplasm are characteristic of oligodendrocytes. At this stage of degeneration, the cytoplasm becomes coarse and grainy as the integrity of the cytoplasmic constituents diminishes.

At the ultrastructural level, the cytoplasm and nucleoplasm of the majority of dying cells were far too coalesced to permit even the most tentative identification. Figure 7 shows a cell in an advanced stage of glial cell death. Numerous mitochondria and Golgi bodies can still be discerned, although they are now extremely bloated and the cytoplasm itself is condensed. Out- lines of long strands of endoplasmic reticulum are also evident. The nucleus is completely condensed and the nuclear membrane has disappeared. These features, although in considerable disarray, are compatible with those of an oligodendrocyte. A cell in a similar stage of degeneration shows apparent continuity with a myelin sheath (Fig. 8). Both the inner tongue of the myelin sheath and the glial cell cytoplasm are in the same plane of focus and are similar in density. This strongly suggests that the dying cell has produced this myelin sheath. Electron-dense particles similar in size to ribosomes appear singly or in clusters throughout the cytoplasm of this cell. These ribosome-like clusters are frequently observed in dying cells at this stage. In Figure 9, a

Figure 4. Twenty day jp mouse spinal cord. $\times 11,400$. An oligodendrocyte is actively ensheathing 2 axons $(A)$. Note the normal chromatin distribution around the nuclear membrane (arrowheads), typical eccentric nucleus, thin strands of endoplasmic reticulum (ER), and Golgi apparatus $(G)$. This oligodendrocyte is similar in morphology to the myelinating oligodendrocytes found in normal spinal cord tissue.

Figure 5. Twenty day jp mouse spinal cord. $\times 23,500$. Oligodendrocyte in an early stage of deterioration. Note the polarization of the chromatin in the nucleus (arrow). The cytoplasmic organelles appear to be normal and are characteristic of immature oligodendroglia. These features include numerous stacks of Golgi apparatus $(G)$, stacks of rough endoplasmic reticulum $(E R)$, several membranous bodies $(M B)$, and numerous free ribosomes. 


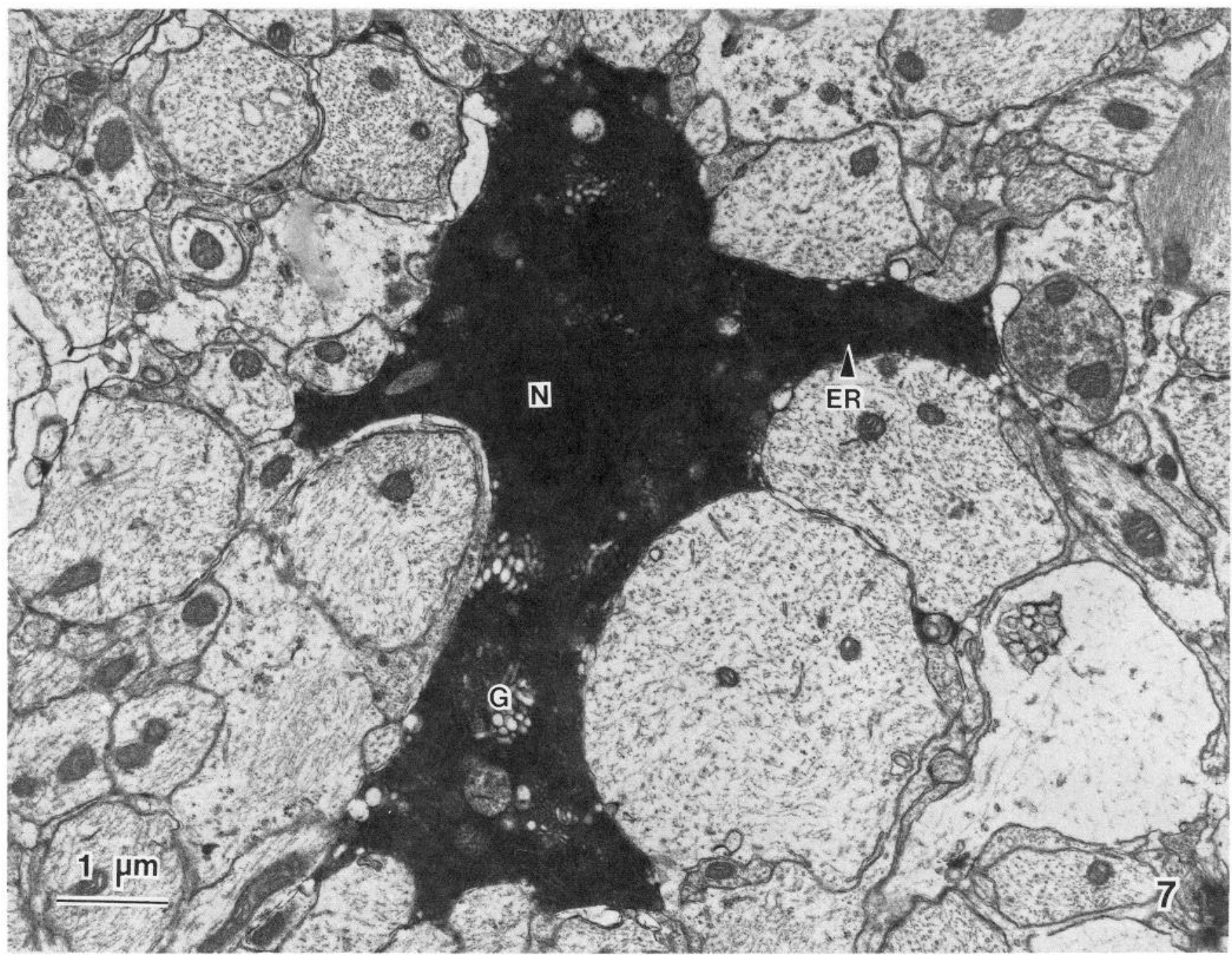

Figure 7. Twenty day jp mouse spinal cord. $\times 16,200$. A cell in an advanced stage of dying. The remnants of many cytoplasmic organelles characteristic of oligodendrocytes are evident ( $G$, Golgi apparatus; $E R$, endoplasmic reticulum). The nucleus $(N)$ is condensed, and the nuclear membrane is no longer visible.

microglial cell engulfs a dead cell mass in a 16-d-old jp spinal cord. Such unidentifiable cellular debris is commonly found in jp CNS tissues. Intermediate filaments, glycogen granules, and other ultrastructural features typical of astrocytes have not yet been observed in dying cells.

\section{Discussion}

Our study of glial cell death in jp mouse CNS led to the following conclusions. First, that glial death in the 3 white matter tracts we studied was significantly greater in jp than in normal animals at specific ages of development; second, that the numbers of dying glia increased in a given tract at a time coincidental with oligodendroglial proliferation and the onset of myelination in that region; third, that the dying glial cells were oligodendrocytes; last, that oligodendroglial cell death proceeded in an orderly pattern, with nuclear abnormalities occurring before those in the cytoplasm.

\section{Time of cell death}

Other investigators have described pyknotic glia in normal CNS at various ages and in various brain regions (Korr, 1980; Pannese and Ferranini, 1967; Smart and Leblond, 1961; Sturrock, 1979). Neuroglial cell losses during development vary among species and between brain regions studied but generally are less than
$1.5 \%$ of the total glial population (Pannese and Ferranini, 1967). In this study, between 0.5 and $2.7 \%$ of the glia in normal CNS tissues were pyknotic.

Analysis of our data showed that the amount of glial cell death in jp spinal cord, optic nerve, and CC was abnormally high. Our first clue in the identification of the dying cell population was the fact that increased glial cell death occurred at different times in each of the $3 \mathrm{CNS}$ areas studied. Increased glial death was observed by postnatal day 4 in jp spinal cord (the earliest age included in this study), by day 12 in jp optic nerve, and, last, by day 16 in jp corpus callosum. This time course parallels that of normal oligodendroglial proliferation and the onset of myelination in each tissue (Skoff, 1976; Sturrock, 1980; Tennekoon et al., 1980). The high percentage of dying glial cells at times coincident with oligodendroglial proliferation and myelination in 3 different tissues strongly suggested that the oligodendrocytes might be dying. We examined thin sections for necrotic cells with recognizable cytoplasmic constituents in order to establish the identity of the dying cell population.

\section{Identification of dying cells}

The cytoarchitecture of the immature oligodendrocyte is uniquely different from that of the developing astrocyte or microglial cell (Mori and Leblond, 1970; Peters et al., 1976; Skoff et al., 1976). 


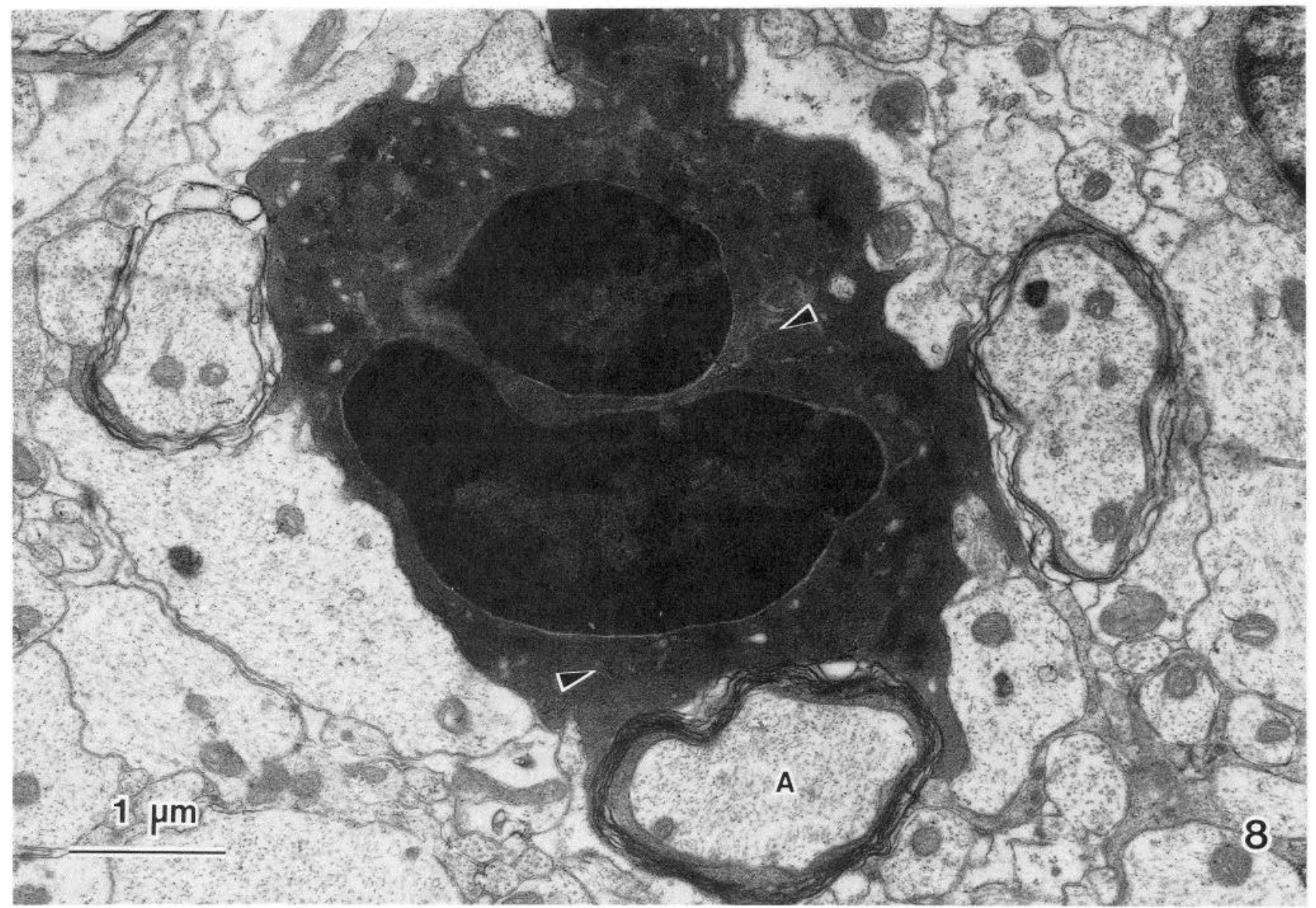

Figure 8. Twenty day jp mouse spinal cord. $\times 21,000$. An oligodendrocyte in an advanced stage of dying. The cytoplasmic organelles are largely disrupted. Identification of the cell would be difficult, except for its continuity with the sheath surrounding the axon $(A)$. Arrowheads indicate clusters of electron-dense particles similar in size to ribosomes.

Classification of the neuroglial cell type can often be made based on morphological criteria alone, provided that a sufficient amount of cytoplasm is examined. In the majority of dying cells, the disruption and deterioration of cytoplasmic organelles makes identification difficult. The cytoplasm of cells in the early stages of pyknosis is, for the most part, intact, and these cells have the morphology of oligodendrocytes (e.g., Fig. 5). Characteristic features of oligodendrocytes seen in dying cells include cisterns of rough endoplasmic reticulum, which are often stacked, prominent Golgi bodies, occasional microtubules, and numerous free ribosomes and mitochondria. The cytoplasm is more electron dense than that of an astrocyte and contains an eccentric nucleus with heterochromatin distributed around the nuclear membrane. Occasionally, processes of dying cells with ensheathed axons are seen. Our impression is that these sheaths are of less than normal thickness. These ultrastructural observations indicate that the majority of dying cells observed in jp CNS tissues are oligodendroglia. Necrotic cells with intermediate filaments or glycogen granules typical of astroglia have not been found. Dying cells tend to be larger than microglia and do not contain the cytoplasmic lipid droplets, inclusions, or the long, vermicular endoplasmic reticulum that are distinguishing features of the microglial cell.

Observations at the light level indicate that approximately $10 \%$ of the total jp glial cell population is dying in $3 \mathrm{CNS}$ regions that should be undergoing active myelination. Since only about $50 \%$ of normal CNS glial cells are oligodendroglia, we estimate that close to $20 \%$ of this cell population is dying at any one time in the jp animal. This figure most likely underestimates the number of sick oligodendroglia since early degenerative changes are not visible at the light level. The death of more than $20 \%$ of the oligodendrocyte pool must have important consequences on myelination in jp animals.

\section{Biochemical changes in jp}

We have presented evidence that large numbers of oligodendrocytes in jp brains die prematurely. A shortened life span is likely to prevent jp oligodendrocytes from forming myelin sheaths of normal thickness. This fundamental explanation for the jp myelin deficit may also explain defects in production and assembly of jp CNS myelin components demonstrated in biochemical studies. The amount of myelin basic protein (MBP) produced by isolated jp ribosomes is either relatively normal (Carnow et al., 1984) or reduced to roughly half of normal levels (Campagnoni et al., 1984). However, when MBP is measured in jp brain homogenates, it is reduced by roughly $95 \%$ (Jacque et al., 1983; Kerner and Carson, 1984; Zimmerman and Cohen, 1979). Isolated ribosomes from jp brain are therefore able to synthesize substantially greater amounts of basic proteins than are actually incorporated into jp myelin (Campagnoni et al., 1984; Carnow et al., 1984). In addition to the reductions in MBP, the myelin marker enzyme $2^{\prime}, 3^{\prime}$-cyclic nucleotide $3^{\prime}$-phosphohydrolase is also at very low levels in jp animals (Kerner and Carson, 1984). Proteolipid protein, a major component of CNS myelin, is apparently entirely absent (Kerner and Carson, 1984). These biochemical analyses indicate oligodendroglial defects involving both synthesis and assembly of myelin components. The present study suggests that the abnormal process- 


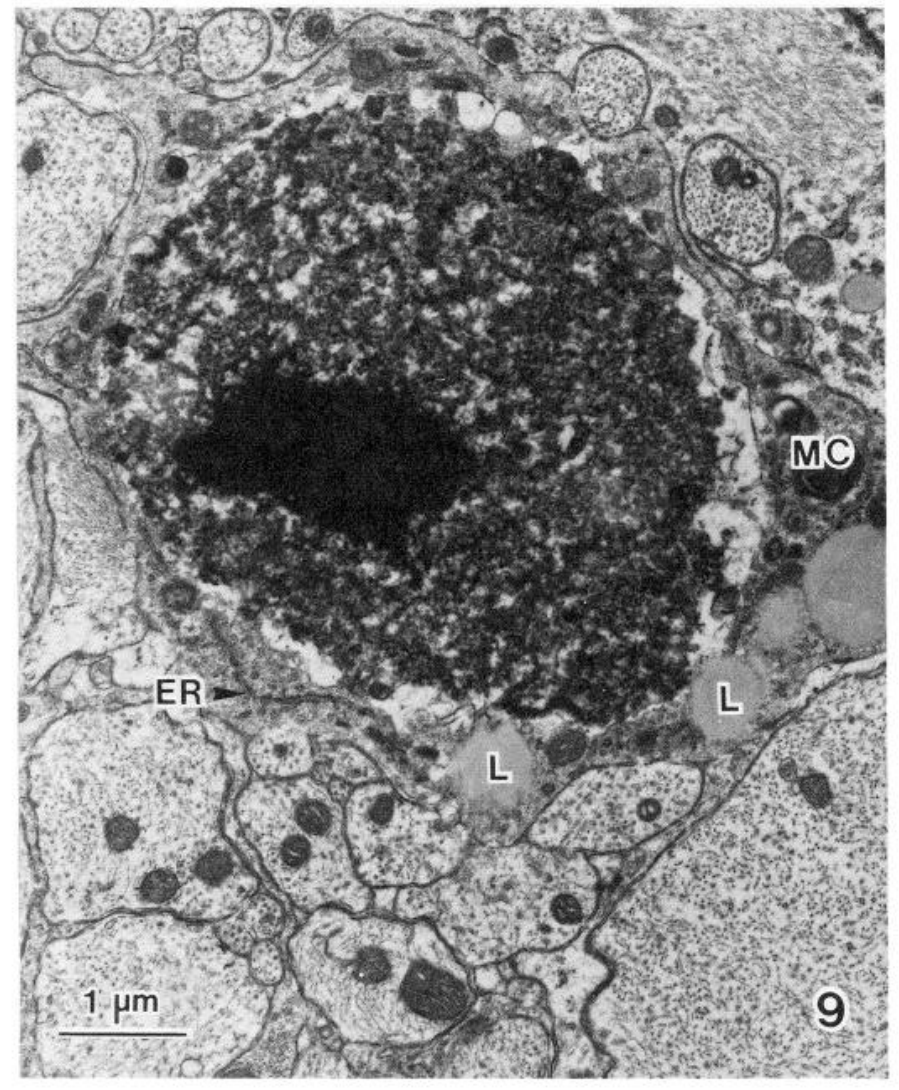

Figure 9. Sixteen day jp mouse spinal cord. $\times 15,000$. A microglial cell $(M C)$ with many lipid droplets $(L)$ and long, stringy endoplasmic reticulum $(E R)$ engulfing a dead cell mass. The dead cell has no recognizable cytoplasmic features.

ing of myelin membranes in jp may be due to a more fundamental problem. Oligodendrocytes that are dying prematurely may be unable to generate new structural components or to maintain those already established. The paucity of cells with cytoplasmic features of mature oligodendrocytes in jp animals is a further indication that this cell line does not differentiate appropriately.

\section{Etiology of the jp defect}

$A$ basic and as yet unresolved question concerns identification of the cell that is the primary target of the jp defect. Studies of female jp carriers show that jp axons are able to support normal myelination. Individual axons in the CNS of jp heterozygotes exhibit areas that are alternately myelinated and unmyelinated along their length (Bartlett and Skoff, 1984; Skoff and Montgomery, 1981). If the defect were in the neuron, its axon would be expected to be completely unmyelinated. Additionally, in vitro studies have shown that coculturing jp cerebellum with normal optic nerve results in significantly increased levels of cerebellar myelination (Billings-Gagliardi et al., 1983). This finding suggests that the mutation is not inherent in the neuron but more likely involves one of the macroglial cells. Both jp astrocytes and jp oligodendrocytes have been shown to exhibit a number of abnormalities. Astrocytic fasciculation is markedly increased in optic nerves of jp mice by $2 \mathrm{~d}$ of age, before oligodendrocytes are present (Skoff, 1976), and by $10 \mathrm{~d}$ of age in the CC (R. P. Skoff, unpublished observations). In jp spinal cords, Omlin and Anders (1983) have described astrocytic hyperplasia, abnormal junctional complexes, and premature development of typical orthogonal particle assemblies in astrocyte membranes. Jimpy astrocytes show evidence of a metabolic defect; $\mathrm{K}^{+}$stimulation of oxygen uptake cannot be demonstrated either in vitro or in vivo (Hertz et al., 1980; Keen et al., 1976). This functional defect and the evidence that astrocytic hyperplasia can occur before the onset of myelination substantially support the idea of abnormalities intrinsic to jp astrocytes.

Mature oligodendrocytes in jp mice are decreased by roughly 50\% (Kraus-Ruppert et al., 1973; Meier and Bischoff, 1975; Privat et al., 1972; Skoff, 1976, 1982), yet, surprisingly, their incorporation of ${ }^{3} \mathrm{H}$-thymidine is severalfold higher than in normal animals (Skoff, 1982).

Given their structural and functional irregularities, both oligodendrocytes and astrocytes may contribute to the myelination defect in the jp animal. One possibility is that oligodendrocytes die prematurely because of astrogliosis. The abnormal insinuation of astroglial processes between axons and oligodendrocytes or their myelin sheaths in jp tissues (Omlin and Anders, 1983) creates a physical barrier that may prevent myelination by disrupting normal cellular contacts. However, there is no evidence that oligodendrocytes require continued axonal contact for survival. In vitro oligodendrocytes elaborate myelin-like membranes in the seeming absence of neurons (Bradel and Prince, 1983; Sarlieve et al., 1983). This differs from the situation in the normal PNS, where the myelinating Schwann cell depends on continued axonal contact for maintenance of myelin sheaths (Weinberg and Spencer, 1978). Occasionally, dying oligodendrocytes in jp tissues appear to be continuous with myelinated axons (Fig. 8). Therefore, if astrocytes do interfere with myelination of jp axons, there must be an additional factor(s) involved in the premature cell death. Another possibility is that the primary defect lies within the oligodendrocyte itself. Other investigators have observed jp axons surrounded by MBP-positive oligodendroglial processes that do not develop into myelin sheaths (Omlin and Anders, 1983). This implies an essential inability of the jp oligodendroglial cell to produce and sustain normal myelin membranes, possibly because of their premature death. Our laboratory has recently compared primary cultures of jp and normal mouse brains. Neurons are not present in these cultures. The majority of oligodendrocytes from normal brains elaborate large, membranous sheets that stain positively for MBP and galactocerebroside. Most cells in jp cultures do not express either of these myelin markers. Staining is largely confined to cell processes and jp cultures exhibit large numbers of dying cells, mimicking the in vivo situation (Bartlett et al., 1985; Knapp et al., 1985). The relatively poor differentiation of jp oligodendrocytes in vitro supports the idea that the jp defect is inherent in one of the macroglial cells.

\section{Patterns of cell death}

Jimpy oligodendroglia follow an orderly sequence of degeneration, beginning with chromatin polarization and condensation. Cytoplasmic irregularities follow the initial nuclear changes and include the swelling of both mitochondria and endoplasmic reticulum. The ribosomes detach and aggregate into dense clusters. The nucleus becomes shrunken, dense, and spherical; its membrane swells, breaks down, and eventually disappears. The Golgi apparatus is preserved for a longer period of time than other organelles. In terminal stages, the cell becomes a solid dark mass that is engulfed by neighboring astroglia or microglia. Our impression is that the early phases of deterioration progress quite rapidly since the majority of cells are in terminal stages (Fig. 9). Preliminary autoradiographic studies suggest that at least some oligodendrocytes die within $3 \mathrm{~d}$ of ${ }^{3} \mathrm{H}$-thymidine incorporation.

The pattern of deterioration in jp oligodendroglia appears to be the reverse of that described for "programmed" or natural neuronal cell death. In natural neuronal cell death, the first signs of morbidity occur in the cytoplasm (Chu-Wang and Oppenheim, 1978; Giordano et al., 1980). It is generally accepted that naturally occurring neuronal cell death in mammals results from 
competition among a neuronal population for some limited trophic or maintenance factor (Hamburger and Oppenheim, 1982). This selective elimination is important in the shaping of the CNS and in determining both the numbers of neurons and their relationships to target cells. The possibility that glial cell death may contribute to the overall scheme of CNS development has been largely overlooked, although the phenomenon has been described in several tissucs (Korr, 1980; Pannese and Ferranini, 1967; Smart and Leblond, 1961; Sturrock, 1979; and as described here). The percentage of glial cells that die at a given time point during normal development is less striking than the cell death that occurs in certain neuronal centers. However, considering the time frame of glial death, which extends throughout most of the postnatal development period (Fig. 1), the overall loss of glial cells will be quite high, perhaps even greater than neuronal losses. Since glial death extends throughout development, it may well play a role in the fine tuning of cellular relationships.

The concept of cell death as a controlled event during morphogenesis is applicable to many tissues. Kerr et al. (1972) have used the phrase "apoptosis" to describe a pattern of shrinkage, or condensation necrosis, resulting in focal cell death during development. In their view, such cells have intrinsic "clocks" and are programmed to regress at particular developmental points, subject to fine tuning by hormones and other environmental inputs (Wyllie et al., 1980). Diffusible environmental factors have long been recognized for their role in CNS development. For example, thyroxine controls the rate of cerebellar cell acquisition and maturation of neurons in both cerebral and cerebellar cortices (Balazs, 1974). Oligodendroglial formation and myelination are also dependent on thyroid hormones and corticosteroids (Balazs et al., 1969; Bohn and Friedrich, 1984; Clos et al., 1982). Corticosteroids inhibit cerebral cell formation, most likely by prolonging the cell cycle (Balazs, 1974).

Our data show that there is a low level of glial death during normal CNS development and that this level may vary with time and between brain regions. Oligodendroglia from jp tissues proliferate at abnormally high rates (Skoff, 1982) even as they are dying prematurely, as shown here. Clearly, there is some factor(s) limiting the expansion of this cell population in the normal animal, just as there must be a factor(s) that limits glial cell loss. The large numbers of dying oligodendroglia in jp animals may be due to a misreading or lack of recognition of factors that normally limit the developmental "pruning" of glial numbers. Whether this results from an error intrinsic to the genetic message or from inappropriate environmental input is unknown. The bulk of recent evidence has shifted the focus of our attention away from a primary defect involving the synthesis and/or assembly of specific myelin components. We now think that the defect in jp involves the control of cell division and early oligodendrocyte differentiation.

\section{References}

Balazs, R. (1974) Influences of metabolic factors on brain development. Br. Med. Bull. 30: 126-134.

Balazs, R., B. W. L. Brooksbank, A.N. Davison, J. T. Eayrs, and D. A. Wilson (1969) The effect of neonatal thyroidectomy on myelination in the rat brain. Brain Res. 15: 219-232.

Bartlett, W. P., and R. P. Skoff (1984) Expression of mosaicism in spinal cords of jimpy heterozygous females. Soc. Neurosci. Abstr. 10. 426.

Bartlett, W. P., P. E. Knapp, and R. P. Skoff (1985) Failure of oligodendrocytes from jimpy brain to differentiate in vitro. Soc. Neurosci. Abstr. 11: 1067.

Billings-Gagliardi, S., L. H. Adcock, E. D. Lamperti, G. Schwing-Stanhope, and M. K. Wolf (1983) Myelination of jp, jp-msd, and qk axons by normal glia in vitro: Ultrastructural and autoradiographic evidence. Brain Res. 268: 255-266.

Bohn, M. C., and V. L. Friedrich (1984) Recovery of myelination in rat optic nerve after developmental retardation by cortisol. J. Neurosci. 2: 1292-1298.

Bradel, E. J., and F. P. Prince (1983) Cultured neonatal rat oligodendrocytes elaborate myelin membrane in the absence of neurons. J. Neurosci. Res. 9: 381-392.

Campagnoni, A. T., C. W. Campagnoni, J.-M. Bourre, C. Jacque, and $\mathrm{N}$. Baumann (1984) Cell-free synthesis of myelin basic proteins in normal and dysmyelinating mutant mice. J. Neurochem. 42: 733739.

Carnow, T. B., J. H. Carson, S. W. Brostoff, and E. L. Hogan (1984) Myelin basic protein gene expression in quaking, jimpy, and myelin synthesis-deficient mice. Dev. Biol. 106: 38-44.

Chu-Wang, I.-W., and R. W. Oppenheim (1978) Cell death of motoneurons in the chick embryo spinal cord. I. A light and electron microscopic study of naturally occurring and induced cell loss during development. J. Comp. Neurol. 177: 33-58.

Clos, J., J. Legrand, N. Limozin, C. Dalmasso, and G. Laurent (1982) Effects of abnormal thyroid state and undernutrition on carbonic anhydrase and oligodendroglia development in the cerebellum. Dev. Neurosci. 5: 243-251.

Cowan, W. M., J. W. Fawcett, D. D. M. O'Leary, and B. B. Stanfield (1984) Regressive events in neurogenesis. Science 225: 1258-1265. Giordano, D. L., M. Murray, and T. J. Cunningham (1980) Naturally occurring neuron death in the optic layers of superior colliculus of the postnatal rat. J. Neurocytol. 9:603-614.

Glücksmann, A. (1951) Cell deaths in normal vertebrate ontogeny. Biol. Rev. 26: 59-86.

Hamburger, V., and R. W. Oppenheim (1982) Naturally occurring neuronal death in vertebrates. Neurosci. Comment. 1(2): 39-55.

Hertz, L., G. Chaban, and E. Hertz (1980) Abnormal metabolic response to excess potassium in astrocytes from the jimpy mouse, a convulsing neurological mutant. Brain Res. 181: 482-487.

Jacque, C., A. Delassalle, M. Raoul, and N. Baumann (1983) Myelin basic protein deposition in the optic and sciatic nerves of dysmyelinating mutants quaking, jimpy, trembler, MLD and shiverer during development. J. Neurochem. 41: 1335-1340.

Keen, P., R. H. Osborne, and U. M. M. Pchrson (1976) Respiration and metabolic compartmentation in brain slices from a glia-deficient mutant, the jimpy mouse. J. Physiol. (Lond.) 245: 22-33.

Kerner, A.-L., and J. H. Carson (1984) Effect of the jimpy mutation on expression of myelin proteins in heterozygous and hemizygous mouse brain. J. Neurochem. 43: 1706-1715.

Kerr, J. F. R., A. H. Wyllie, and A. R. Currie (1972) Apoptosis: A basic biological phenomenon with wide-ranging implications in tissue kinetics. Br. J. Cancer 26: 239-257.

Knapp, P. E., and R. P. Skoff (1984) Increased glial cell death in jimpy mouse optic nerve and spinal cord. Soc. Neurosci. Abstr. 10: 428.

Knapp, P. E., W. P. Bartlett, and R. P. Skoff (1985) Production of $\mathrm{MBP}+$ and $\mathrm{GC}+$ membranous sheets by oligodendrocytes in vitro. Soc. Neurosci. Abstr. 11: 1067.

Korr, H. (1980) Proliferation of different cell types in the brain. Adv. Anat. Embryol. Cell Biol. 61: 1-72.

Kraus-Ruppert, R., N. Herschkowitz, and S. Furst (1973) Morphological studies on neuroglial cells in the corpus callosum of the jimpy mutant mouse. J. Neuropathol. Exp. Neurol. 32: 197-203.

Kuwabara, T. (1975) Development of the optic nerve of the rat. Invest. Opthalmol. 14: 732-745.

Meier, C., and A. Bischoff (1975) Oligodendroglial cell development in jimpy mice and controls, an electron-microscopic study in the optic nerve. J. Neurol. Sci. 26: 517-528.

Mori, S., and C. Leblond (1970) Electron microscopic identification of three classes of oligodendrocytes and a preliminary study of their proliferative activity in the corpus callosum of young rats. J. Comp. Neurol. 139: 1-30.

Nussbaum, J. L., N. Neskovic, and P. Mandel (1969) Effect of the jimpy mutation on expression of myelin proteins in heterozygous and hemizygous mouse brain. J. Neurochem. 16:927-934.

Omlin, F. X., and J. J. Anders (1983) Abnormal cell relationships in jimpy mice: Electron microscopic and immunocytochemical findings. J. Neurocytol. 12: 767-784.

Pannese, E., and E. Ferranini (1967) Nuclear pyknosis in neuroglia cells of normal animals. Acta Neuropathol. 8: 309-319.

Peters, A., S. L. Palay, and H. deF. Webster (1976) The Fine Siructure of the Nervous System: The Neurons and Supporting Cells, Saunders, Philadelphia, PA. 
Privat, A., O. Robain, and P. Mandel (1972) Aspects ultrastructuraux du corps calleux chez la souris jimpy. Acta Neuropathol. (Berl.) 21: 282-295.

Sarlieve, L. L., M. Fabre, J. Susz, and J. M. Matthieu (1983) Investigations on myelination in vitro: IV. "Myelin-like" or premyelin structures in cultures of dissociated brain cells from 14-15 day-old embryonic mice. J. Neurosci. Res. 10: 191-210.

Sidman, R. L., M. M. Dickie, and S. H. Appel (1964) Mutant mice (quaking and jimpy) with deficient myelination in the central nervous system. Science 144: 209-211.

Skoff, R. P. (1976) Myelin deficit in the jimpy mouse may be due to cellular abnormalities in astroglia. Nature 264: 560-562.

Skoff, R. P. (1982) Increased proliferation of oligodendrocytes in the hypomyelinated mouse mutant-jimpy. Brain Res. 248: 19-31.

Skoff, R. P., and I. N. Montgomery (1981) Expression of mosaicism in females heterozygous for jimpy. Brain Res. 212: 175-181.

Skoff, R. P., D. L. Price, and A. Stocks (1976) Electron microscopic autoradiographic studies of gliogenesis in rat optic nerve. II. Time of origin. J. Comp. Neurol. 169: 313-333.
Smart, I., and C. P. Leblond (1961) Evidence for division and transformations of neuroglia cells in the mouse brain, as derived from radioautography after injection of thymidine-3H. J. Comp. Neurol. 116: 349-367.

Sturrock, R. R. (1979) A quantitative lifespan study of changes in cell number, cell division and cell death in various regions of the mouse forebrain. Neuropathol. Appl. Neurobiol. 5: 433-456.

Sturrock, R. R. (1980) Myelination of the mouse corpus callosum. Neuropathol. Appl. Neurobiol. 6: 415-420.

Tennekoon, G. I., Y. Kishimoto, I. Singh, G. Nonaka, and J. M. Bourre (1980) The differentiation of oligodendrocytes in the rat optic nerve. Dev. Biol. 79: 149-158.

Weinberg, H. J., and P. S. Spencer (1978) The fate of Schwann cells isolated from axonal contact. J. Neurocytol. 7: 555-569.

Wyllie, A. H., J. F. R. Kerr, and A. R. Currie (1980) Cell death: The significance of apoptosis. Int. Rev. Cytol. 68: 251-306.

Zimmerman, T. R., and S. R. Cohen (1979) The distribution of myelin basic protein in subcellular fractions of developing jimpy mouse brain. J. Neurochem. 32: 817-821. 